\title{
CONSENSUS BASED DATA AggREgATION FOR ENERGY CONSERVATION IN WIRELESS SENSOR NETWORK
}

\author{
Tamal Banerjee, Priyansh Sharma and Shrijana Pradhan \\ Department of Computer Science and engineering, Sikkim Manipal \\ Institute of Technology, India
}

\begin{abstract}
Our planet is abundant with raw data and to monitor the available data properly, processing of the enormous raw data is very vital. One of the key things in development of mankind and the nature is to acquire as much data as possible and to react appropriately in accordance with the studied data. It's nothing but diagnosis of the physical world by studying the data acquired from them in order to take proper measures that can help in treating them better. Large volume of data incurs high energy consumption for its transmission and thus results in decrease of overall network lifetime.

Wireless Sensor Network (WSN) is a collection of multiple sensor nodes that all together forms a network for transmitting data acquired by each sensor node to sink known as Base Station (BS). In hierarchical routing acquired data are sent via relay agents like Cluster Heads (CH). The Cluster Heads must be customised with computations and formulations, which will help in aggregating the gathered data, in order to reduce energy consumption while transmitting the data further in the network while maintaining the data integrity to withhold the significance of every single value in a data set.
\end{abstract}

The proposed work devised a data aggregation technique to reduce the size of data frame in Wireless Sensor Network which will have the following benefits:

- $\quad$ Reduce the amount of data traffic between sensor nodes.

- $\quad$ Reduce overall energy consumption of the network.

- $\quad$ Communicating more data each round between Sensor Heads and finally to Base Station.

- Increase in network lifetime.

The work performs data aggregation at bit level which produces better aggregated data as significance of all the bits are included in the data.

The consensus based data aggregation algorithm is further used to modify Low-Energy Adaptive Clustering Hierarchy (LEACH) protocol for conserving energy and improving the network performance. Various parameters that are taken into consideration for evaluating Proposed Work protocol:

- Operating Nodes per round

- Operating Nodes per Transmission

- Energy Consumed per Transmission

- Average Energy Consumed by a Node per Transmission

- Network Life-Time

\section{KEYWORDS}

Wireless Sensor Network (WSN), Data Aggregation, Cluster Heads (CH), Base Station (BS), Low-Energy Adaptive Clustering Hierarchy (LEACH) protocol 


\section{INTRODUCTION}

Wireless Sensor Network (WSN) is a network of multiple sensors within a physical phenomenon that communicates the collected data wirelessly. These sensors will transmit various data within the network which will be then aggregated to transform these data into reduced form, holding data integrity [8]. This aggregation of data will happen in between the sensor heads and finally to base station during data transmission and final aggregated data will be directly sent to the base station for further actions.

A sensor is a device that translates physical parameters like energy into signals that is electrical energy which are not able to be processed directly. This signal is passed through signal conditioning stage so as to make the signal able to be used further. Analogue-to-digital converter (ADC) is used to convert the signal to digital signal for further visualization, processing and storing. [16]

Wireless sensors have in-built sensing, processing, communicating and storing capabilities. These sensors form a network to analyze physical environments. The sensor nodes communicate with each other as well as to the base station either in single-hop or in multi-hop. In single-hop, each sensor node communicates directly to the base station whereas in multi-hop some sensor nodes also serve as relay agent which collaboratively propagates sensor data towards the base station. [16]

For communicating the data to the base station, LEACH protocol [17] is used. In this protocol, the sensor nodes are grouped into clusters [14] and each cluster is assigned with a cluster head, which will aggregate the data and then will transmit it either via relay agents or directly to the base station. The cluster head is selected based upon the threshold energy found by equation 2 [19] [7].

The sensor nodes are provided with limited power supply and as they are wirelessly deployed so it is very important to conserve the energy as much as possible so as to increase the network lifetime [8]. Thus, the Proposed Work protocol will help in conserving the energy of the network by reducing the data packet size during aggregation to single value which will represent the whole cluster using consensus based data aggregation at cluster head. [3]

Network Lifetime provides the sustainability of the network based upon the network energy calculated by equation 3 [7]. It can be viewed as:

1. First Dead Node: Round number in which the energy of any node in WSN gets exhausted first.

2. Half Dead Node: Round number in which the energy of half of the nodes in WSN gets exhausted.

3. Final Dead Node: Round number in which the energy of all the nodes in WSN gets exhausted. 


\section{LiteratURE SURVEY}

Survey on Data Capturing/Processing/Transmission in WSN [9]:

The survey reveals the importance of data capturing and transmitting it after processing so as to gather important and crucial information from the data and to forward the info ahead.

\section{TH-LEACH: Threshold Value and Heterogeneous Nodes-Based Energy-Efficient LEACH Protocol [2]:}

Energy is required for a sensor node to transmit data. So to improve the lifetime of a cluster which indirectly improves the lifetime of the network, the energy of each sensor node and sensor head must be conserved as performance of wireless sensor network depends upon lifetime of the network that is directly proportional to the energy available to the network.

\section{Energy Conservation in Wireless Sensor Networks by Exploiting Inter-Node Data Similarity Metrics [6]:}

Sensor head transmits data to base station in either single hop or multiple hops. Thus sensor head takes data from sensor nodes and then transmits it after aggregation for better network performance. While accumulating data from different sensor nodes within a same cluster there is a chance of getting duplicate data. Energy can be conserved before data aggregation at sensor head by reducing duplicate data within the same cluster.

Urban Sprawl Analysis of Tripoli Metropolitan City (Libya) Using Remote Sensing Data and Multivariate Logistic Regression Model [13]:

Urban sprawl was analysed using logistic regression model in Metropolitan city (Libya). The expansion of people is quite similar to raw data that sprawls in nature. Logistic regression model validation was conducted using the relative operating characteristic (ROC) method. The validation result was having 0.86 accuracy rate. The results indicated that the logistic regression model is effective in explaining urban expansion and thus it was used in finding threshold for humidity data sensed by the wireless sensors deployed in soil.

\section{Problem Definition}

Data nowadays are enormous and to monitor the available data properly, processing of the huge data is very vital. These data takes a lot of time to get transmitted and thus delays the respond time towards the incoming data. Large volume of data incurs high energy consumption that results in decrease of network lifetime. Also during the transmission of the data via cluster heads, data integrity is very hard to achieve as the significance of every single value in a data set is tough to maintain. This leads to in-accuracy in data transmission.

\section{Proposed Solution Strategy}

Data needs to be transmitted on time and there should be no or less data traffic within the network so that data can be responded accordingly to take corresponding action. Thus data aggregation is required within the network for conserving the energy of the network which in order increases the network lifetime and to improve the performance of the network. So aggregation part of LEACH protocol is modified so as to reduce data packet size by using consensus based data aggregation technique at cluster head, which will then reduce the energy 
consumption for transmitting the data further in the network and thus will result in decrease of overall energy consumption of the network and will increase network lifetime.

\section{Methodology}

In this modified LEACH protocol, the consensus based data aggregation technique used at cluster heads is given as follows:

Step 0: Start

Step 1: Read Dataset.

Step 2: for $i$ in range 1 to (length of dataset):

Convert each decimal data to binary

Step 3.1: Apply machine learning upon the dataset by forming training and testing dataset.

Step 3.2: threshold $=60 \%$ (Based upon Logistic Regression over humidity data set)

Step 4: for $i$ in range (length of data):

than threshold)

Check (value at position[i] for all the bits in data is greater

\section{If(true): \\ Store 1 at ith binary position in final binary result \\ Else: \\ Store 0 at ith binary position in final binary result}

Step 5: Convert final binary to decimal

Step 6: Take final decimal as representative for entire dataset/cluster.

Step 7: Forward final decimal value from one cluster head to another or to base

station.

Step 8: End.

The proposed algorithm installed at each relay nodes aggregates and transfer data ahead. In the given consensus based data aggregation, the data will be collected by the sensors deployed in the physical environment. Here the sensed data is humidity data [21] that describes the topological condition. Now cluster formation will take place via LEACH protocol [20]. The data collected in a cluster will be forwarded to cluster head. At cluster head all the data will be converted to binary. Now logistic regression will help to find the threshold, using equation 1, for aggregation of all the data at sensor head [1].

In Logistic Regression all the data will be trained first. Then all the data will be plotted based upon sigmoid function, which plots the data between 0 to 1 giving the probability of the dataset. Then midway the S-curve, that is obtained by applying sigmoid function, will be divided and the probability of data lying on curve after midway will be considered as 1 and rest will be 0 . Thus, this will give the threshold.

$$
y=\frac{1}{1+e^{-x}}
$$

equation 1

where, $\mathrm{y}$ gives the value on $\mathrm{S}$-curve and $\mathrm{x}$ are the points for which values are to be taken.

Based upon the threshold, all the data will be aggregated to single value. At every binary position for all the data simultaneously, if the percentage of 1 present at that binary position is more than that of the threshold then put 1 at the same binary position in the final aggregated value. In this way continue till all the binary positions are traversed. 
Finally, a single value will be obtained at the sensor head which will represent the overall cluster holding the integrity and significance of each data in the cluster [8]. Likewise, the cluster heads, sensor nodes will transfer data finally to base station and at base station a single value will be obtained with the help of consensus based data aggregation that will be representing the entire network.

In LEACH protocol there are 2 phases [20]. First one is Set-Up phase, where the protocol decides the node which is applicable for the next cluster head in the corresponding area. Protocol also clusters the nodes of the corresponding area to the cluster head.

Process: Protocol selects the node for the cluster head on the basis of Threshold energy. Threshold energy calculated on basis of following formula :

$$
\mathrm{t}(\mathrm{n})=\left(\mathrm{p} /\left(1-\mathrm{p}^{*}(\bmod (\mathrm{r}, 1 / \mathrm{p}))\right)\right)
$$

Where,

$\mathrm{t}$ - Threshold Energy

$\mathrm{p}-$ No. of nodes desired per cluster

n- No. of nodes

$\mathrm{r}-$ Round No.

Every wireless sensor node has their own energy for sending and receiving the data. The node having energy smaller than this Threshold energy will be applicable to be a cluster head at once. In this way, every node will be cluster head, one at a time and the node which is previously allowed for the cluster head will not be allowed for the cluster head in future until every node gets a chance to be cluster head once.

Second phase is Steady-State Phase, Transfer and Reception of data takes place and energy dissipates continuously with transfer of data. [4] The Energy Dissipation mainly depends on the distance between the adjacent nodes where transfer is taking place [1]. It is calculated by the following formula [7];-

$$
\mathrm{Ex}=\text { Elect } * \mathrm{k}+\text { Amp }^{*} \mathrm{k} * \mathrm{SN}(\mathrm{i}) \cdot \text { Dist }^{\wedge} 2
$$

where,

Elect: Energy consumed to open and close the packet at the node.

Ex: Energy consumed units in Joules/bit

K: Total Energy units in bits

Amp: amount of energy spent by the amplifier to transmit the bits

Dist: distance between the adjacent nodes

\section{IMPLEMENTATION DETAILS}

Humidity data was used during simulation which helped in understanding the topological condition of a particular area. Out of the entire dataset available, 100 random humidity data are used for simulation and performing the comparison between actual and modified LEACH protocol. For analysing and responding in accordance to the sensed humidity value, the humidity data is aggregated by modifying LEACH protocol using consensus based data aggregation so as 
International Journal of Distributed and Parallel Systems (IJDPS) Vol.11, No.1/2/3/4/5, September 2020

to conserve energy of the sensors used for sensing humidity. The proposed work was evaluated against LEACH protocol.

\section{Data Availability Statement}

The humidity data used to support the findings of this study have been deposited in the KAGGLE repository.

(Dataset Link: https://www.kaggle.com/yungbyun/sensor-data).

Accessed on-(23/09/2019, 9:27:59 am).

\section{Sample dataset}

Out of the overall humidity data, 20 humidity data are taken as a sample.

Table 1: Sample Dataset

\begin{tabular}{|l|l|l|l|}
\hline 1)33.06045426 & 2)33.09548316 & 3)33.08046737 & 4)33.10548665 \\
\hline 5$) 33.10548169$ & 6)33.10047989 & 7)33.12549354 & 8)33.13049525 \\
\hline 9)33.14549582 & $10) 33.14549582$ & $11) 33.12048705$ & $12) 33.15549928$ \\
\hline 13$) 33.14549582$ & $14) 33.15549928$ & $15) 33.15550367$ & $16) 33.1805119$ \\
\hline 17$) 33.18550949$ & $18) 33.20051836$ & $19) 33.20051836$ & $20) 33.20551995$ \\
\hline
\end{tabular}

\section{EXPERIMENTAL RESUlts}

The proposed algorithm was carried out considering following simulation parameters.

\section{Simulation parameters}

Number of nodes : 100

Area : $(70 \times 70) \mathrm{m} 2$

Simulation time (s) : $\{50,100,150,200,250,300\}$

Data packet size (B) : 2000

Deployment : Randomly uniformly distributed

Mobility : Static

Radio module : CC2420.txt

Eelect : $50 \mathrm{~nJ} / \mathrm{bit}$

Eamp : $0.00134 \mathrm{pJ} / \mathrm{bit} / \mathrm{m} 4$

Eaggr : $5 \mathrm{~nJ} / \mathrm{bit} / \mathrm{signal}$

Results after applying the consensus based data aggregation algorithm over LEACH Protocol:

\subsection{Operating Nodes per Rounds}

Operating nodes serves the purpose of acquiring sensor data and transmitting the data through the sensor network. The result in Table 2 shows the rounds up to which the nodes remain operating by being able to acquire and transmit sensor data. 
International Journal of Distributed and Parallel Systems (IJDPS) Vol.11, No.1/2/3/4/5, September 2020

Table 2: Operating Nodes per Round

\begin{tabular}{|c|c|c|}
\hline Round & $\begin{array}{c}\text { Operating Nodes } \\
\text { (Modified LEACH) }\end{array}$ & $\begin{array}{c}\text { Operating Nodes } \\
\text { (LEACH) }\end{array}$ \\
\hline 1000 & 100 & 100 \\
\hline 2000 & 100 & 9 \\
\hline 3000 & 76 & 0 \\
\hline 4000 & 54 & 0 \\
\hline 5000 & 39 & 0 \\
\hline 6000 & 22 & 0 \\
\hline 7000 & 13 & 0 \\
\hline 8000 & 6 & 0 \\
\hline 9000 & 1 & \\
\hline
\end{tabular}

Below graph shows the comparison among actual and Proposed Work protocol regarding the nodes being able to operate with respect to number of rounds.

Round: Round is time slot for transfer of data from one node to base station (via number of cluster heads and finally to base station).



Figure 1: Operating Nodes per round (LEACH)

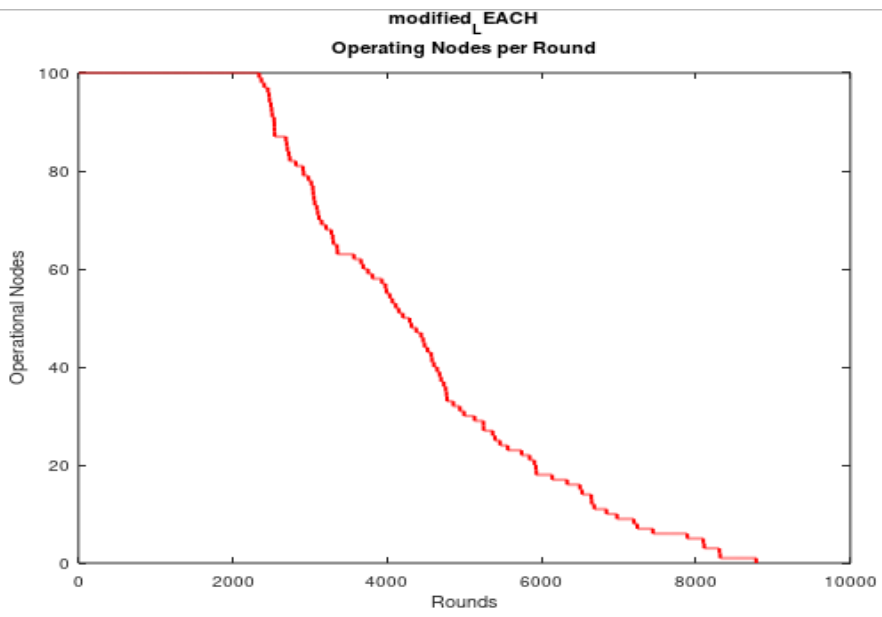

Figure 2: Operating Nodes per round (Proposed Work) 
Operating Nodes per round (LEACH) :- In Figure 1, shows the number of operating nodes per round that means in one round how many operating nodes are working. It also gives the information about how many nodes are becoming dead in each round. The other information is that after how many rounds, all the operating nodes will become dead (Life Time of the protocol on the basis of number of rounds). Operating Nodes per round (Proposed Work):- In Figure 2 shows the result after applying consensus based data aggregation at sensor head in LEACH protocol.

\section{Advantage:}

It was observed that in Figure 1, the energy of all the operating nodes is getting exhausted by 2500 rounds whereas In Figure 2, all operating nodes are surviving till 8000+ rounds making the proposed technique more efficient than that of LEACH. Network Life time Gain Percentage per round $=68.75 \%$

\subsection{Operating Nodes per Transmission}

The result in Table 3 shows the number of nodes that remains operating by being able to acquire and transmit sensor data per transmission.

Table 3: Operating Nodes per Transmission

\begin{tabular}{|c|c|c|}
\hline Transmission & $\begin{array}{c}\text { Operating Nodes } \\
\text { (Modified LEACH) }\end{array}$ & Operating Nodes (LEACH) \\
\hline 1000 & 100 & 100 \\
\hline 2000 & 100 & 0 \\
\hline 3000 & 75 & 0 \\
\hline 4000 & 53 & 0 \\
\hline 5000 & 34 & 0 \\
\hline 6000 & 17 & 0 \\
\hline
\end{tabular}

Figure 3 and 4 shows the comparison among actual and Proposed Work protocol regarding the nodes being able to operate per transmission.

Transmission: Transmission is the time slot for transfer of data from one node to base station or one node to cluster head or cluster head to cluster head or cluster head to base station.

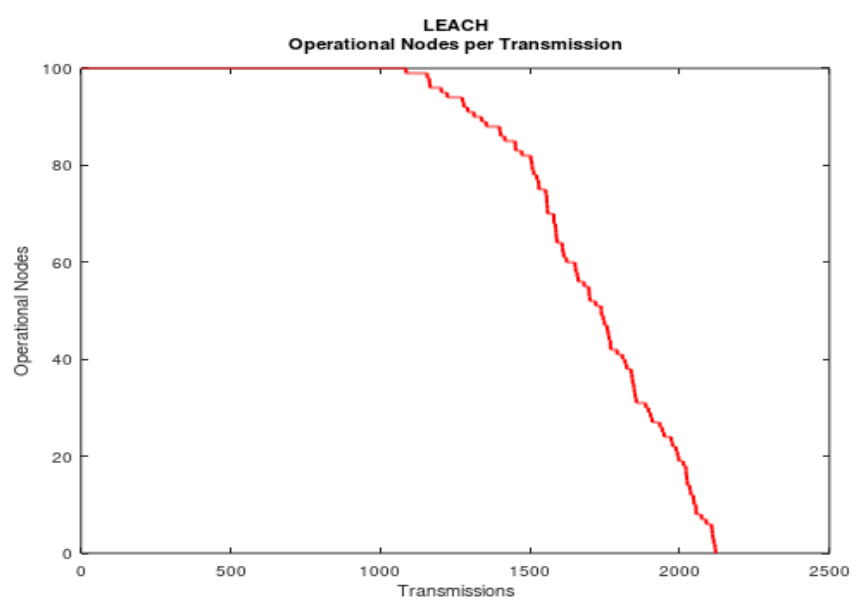

Figure 3: Operating Nodes per Transmission (LEACH) 




Figure 4: Operating Nodes per Transmission (Proposed Work)

Operating Nodes per Transmission (LEACH): - Figure 3 gives the information about the number of operating nodes per transmission that means in a transmission how many operating nodes are working. It also gives the information about how many nodes are becoming dead in which transmission. The other information is that after how many transmissions, all the operating nodes will become dead (Life Time of the protocol on the basis of number of transmissions).

Operating Nodes per Transmission (Proposed Work): - In Figure 4,it gives the same information as Figure 4 gives but this gives the information about the modified leach (i.e. consensus based leach).

\section{Advantage:}

It was observed that in Figure 3 (LEACH protocol), all the operating nodes were dead before 2500 transmissions whereas in Figure 4 (Proposed Work), all operating nodes are becoming dead after 6000 transmissions. Network Life time Gain Percentage per transmission $=58.33 \%$

\subsection{Energy consumed per transmission}

Energy consumed denotes the energy associated to a node, after consuming certain amount of energy from available energy of the node, for acquiring, aggregating and transmitting the sensor data. The following result shows the energy consumed by the network per transmission.

Table 4: Energy consumed per transmission

\begin{tabular}{|c|c|c|}
\hline Transmission & Energy(J) (Modified LEACH) & Energy(J) (LEACH) \\
\hline 1 & 0.041413 & 0.116246 \\
\hline 201 & 0.0984561 & 0.111358 \\
\hline 401 & 0.0513913 & 0.114959 \\
\hline 601 & 0.0129045 & 0.135568 \\
\hline 801 & 0.0298366 & 0.138249 \\
\hline 1001 & 0.039545 & 0.126957 \\
\hline 1201 & 0.0422535 & 0 \\
\hline 1401 & 0.0852474 & 0 \\
\hline 1601 & 0.0447934 & 0 \\
\hline 1801 & 0.0155833 & 0 \\
\hline 2001 & 0.0286065 & 0 \\
\hline 2201 & 0.0627182 & 0 \\
\hline
\end{tabular}


International Journal of Distributed and Parallel Systems (IJDPS) Vol.11, No.1/2/3/4/5, September 2020

Figure 5 and 6 shows the comparison between LEACH and Proposed Work protocol regarding the network consuming energy per transmission.

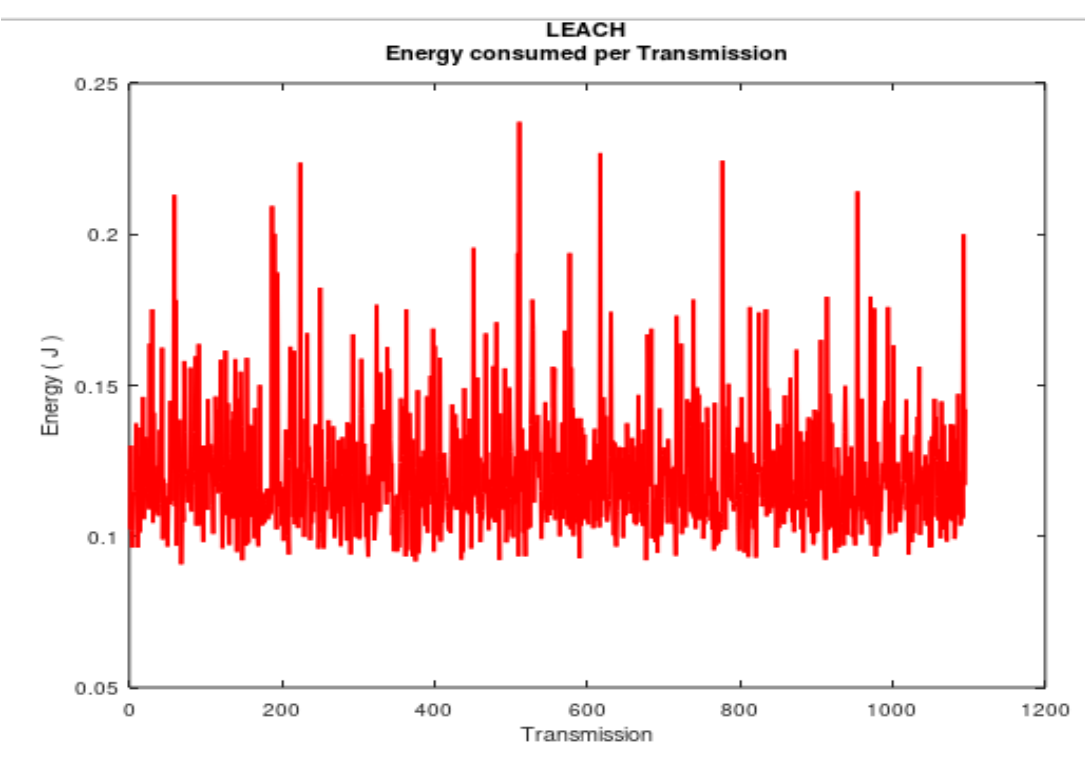

Figure 5: Energy Consumed per Transmission (LEACH)

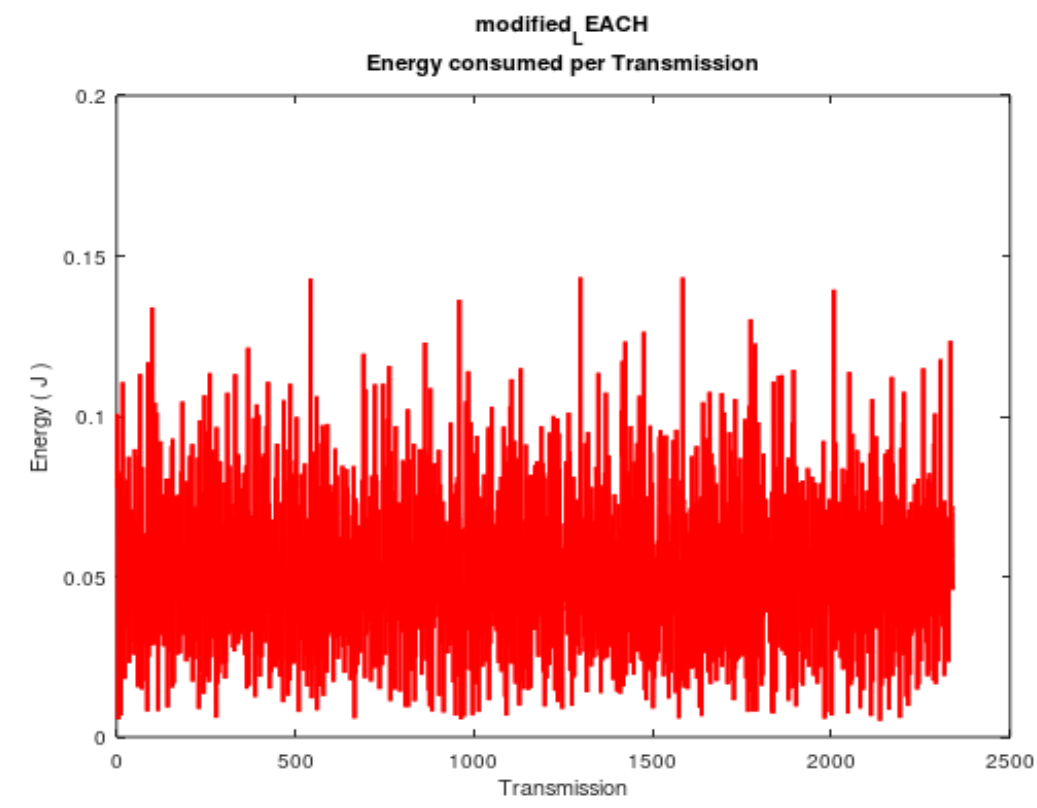

Figure 6: Energy Consumed per Transmission (Proposed Work)

Every node has their own energy to do their works. For transferring and reception of data, some energy will be consumed.

Energy Consumed per Transmission (LEACH):- Figure 5 shows the energy consumption of the nodes at every transmission in LEACH protocol.

Energy Consumed per Transmission (Proposed Work):- Figure 6 shows the energy consumption of the nodes at every transmission in modified LEACH protocol. 
International Journal of Distributed and Parallel Systems (IJDPS) Vol.11, No.1/2/3/4/5, September 2020

Energy consumption basically depends on the distance between the adjacent nodes. It was calculated by equation 3 :

$$
\mathrm{Ex}=\text { Elect } * \mathrm{k}+\text { Amp } * \mathrm{k} * \mathrm{SN}(\mathrm{i}) \cdot \text {.Dist2 }
$$

equation 3

\section{Advantages:}

It was observed that in Figure 5 (LEACH protocol), energy consumption of the node starting in the range from 0.1 to 0.15 whereas in Figure 6 (Modified LEACH protocol), energy consumption of the node starting in between the range from 0.01 to 0.05 .

In the Figure 5 (LEACH protocol), energy consumption of the node was ending in between the range from 0.13 to 0.22 whereas in Figure 6 (Modified LEACH protocol) energy consumption of the node was ending before 0.15 .

The aggregation of the waves in Figure 6 is more than the aggregation of the waves in Figure 5. This implies that at some instant of time, more transmissions can take place with less amount of energy.

\subsection{Average Energy consumed by a node per transmission}

The following result shows the overall average energy consumed by the network per transmission.

Table 5: Average Energy consumed by a Node per Transmission

\begin{tabular}{|c|c|c|}
\hline Transmission & Energy $(\mathbf{J})($ Modified LEACH) & Energy $(\mathbf{J})(\mathbf{L E A C H})$ \\
\hline 1 & 0.041413 & 0.116246 \\
\hline 201 & 0.0984561 & 0.111358 \\
\hline 401 & 0.0513913 & 0.114959 \\
\hline 601 & 0.0129045 & 0.135568 \\
\hline 801 & 0.0298366 & 0.138249 \\
\hline 1001 & 0.039545 & 0.126957 \\
\hline 1201 & 0.0422535 & 0 \\
\hline 1401 & 0.0852474 & 0 \\
\hline 1601 & 0.0447934 & 0 \\
\hline 1801 & 0.0155833 & 0 \\
\hline 2001 & 0.0286065 & 0 \\
\hline 2201 & 0.0627182 & 0 \\
\hline
\end{tabular}

Figure 7 shows the comparison among actual and Proposed Work protocol regarding the average energy consumed by the entire network per transmission. 
International Journal of Distributed and Parallel Systems (IJDPS) Vol.11, No.1/2/3/4/5, September 2020

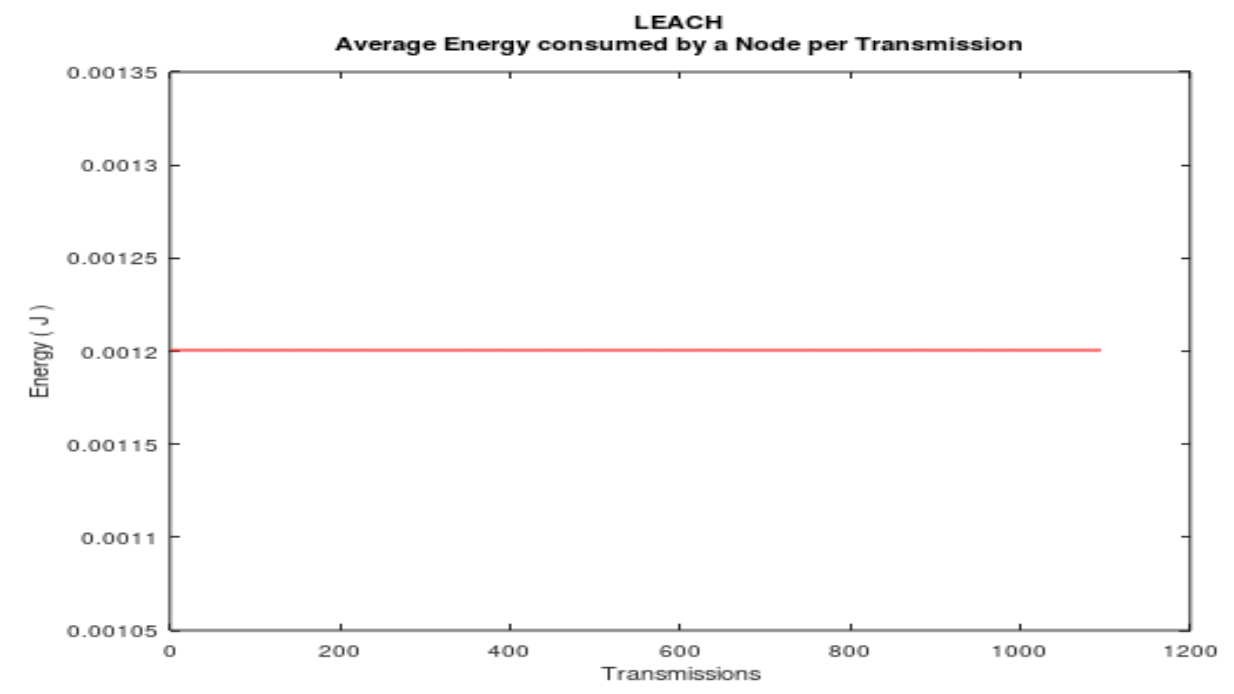

Figure 7: Average Energy Consumed by a Node per Transmission (LEACH)

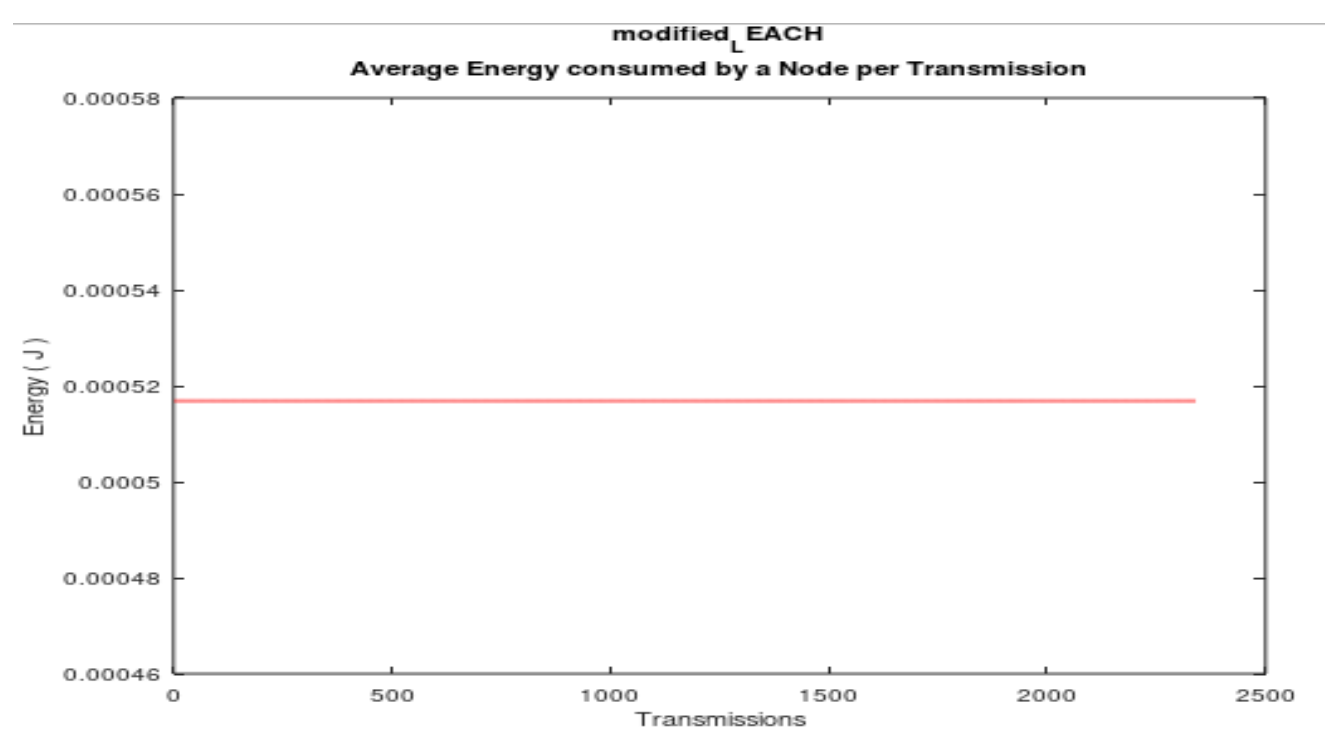

Figure 8: Average Energy Consumed by a Node per Transmission (Proposed Work)

Figure 7 (LEACH protocol) shows the average of the energy consumed by the nodes. Figure 8 (Modified LEACH protocol) shows the average of the energy consumed by the nodes.

\section{Advantage:-}

In Figure 7, Average energy consumed $=0.0012$.

In Figure 8, Average energy consumed $=0.00052$.

This gives a significant amount of conservation in energy consumption while using Proposed Work protocol using consensus based data aggregation.

Increase in average Energy consumption Percentage $=56.667 \%$ 


\subsection{Network Life-Time}

The lifetime of a network is determined by the quantity of nodes that remain operational over period of time. First node dead is considered as the round at which the first node of the network uses all its available energy and gets exhausted and thus fails to operate further. Similarly, Half node dead is considered as the round at which half of the nodes of the entire network uses all their available energy and gets exhausted and thus fails to operate further. All nodes dead is considered as the round at which every node in the entire network uses all their available energy and thus the network becomes dead as not a single node in the network is capable to operate further.

Table 6 and 7 shows the comparison among actual and Proposed Work protocol regarding the lifetime of the entire network upon parameters like first node dead, half nodes dead and all nodes dead.

Table 6: Network Life-Time (LEACH)

\begin{tabular}{|c|c|}
\hline $\begin{array}{l}\text { LEACH } \\
\text { protocol }\end{array}$ & Round \\
\hline First Node Dead & 1140 \\
\hline Half Nodes Dead & 2183 \\
\hline All Nodes Dead & 2594 \\
\hline
\end{tabular}

Table 7: Network Life-Time (Proposed Work)

\begin{tabular}{|c|c|}
\hline $\begin{array}{l}\text { Proposed Work } \\
\text { protocol }\end{array}$ & Round \\
\hline First Node Dead & 1410 \\
\hline Half Nodes Dead & 4177 \\
\hline All Nodes Dead & 8518 \\
\hline
\end{tabular}

As shown in Table 6, First Node is becoming dead at round number 1140 and half of the nodes are becoming dead at round number 2183 and all nodes are becoming dead at round number 2594.

As shown in Table 7, First Node becoming dead at round number 1410 and half of the nodes are becoming dead at round number 4177 and all nodes are becoming dead at round number 8518 .

Thus Proposed Work protocol gives an advantage over total network lifetime by decreasing the energy consumption of the entire network.

Network lifetime percentage increased $=69.94 \%$

\subsection{Data aggregation at each round}

Data aggregation is done by consensus based data aggregation algorithm applied during modifying the LEACH protocol.

Table 8 shows the cluster and cluster head formation at each round along with the values of each node inside the cluster and the final aggregated value found after data aggregation at each round. 
International Journal of Distributed and Parallel Systems (IJDPS) Vol.11, No.1/2/3/4/5, September 2020

Table 8: Aggregated data at base station per round

\begin{tabular}{|c|c|c|c|c|c|}
\hline Round & $\begin{array}{l}\text { Cluster } \\
\text { Head/s }\end{array}$ & Nodes & Values & $\begin{array}{l}\text { Aggregated } \\
\text { Value }\end{array}$ & $\begin{array}{c}\text { Final aggregated } \\
\text { Value at base station }\end{array}$ \\
\hline \multirow[t]{2}{*}{1} & 2 & $\begin{array}{c}3 \\
8, \\
9, \\
10, \\
14, \\
16\end{array}$ & $\begin{array}{c}33.08046737, \\
33.13049525, \\
33.14549582, \\
33.14549582, \\
33.15549928, \\
33.1805119\end{array}$ & 33.125 & 33.125 \\
\hline & 20 & $\begin{array}{l}1, \\
4, \\
5, \\
6, \\
7, \\
11, \\
12, \\
13, \\
15, \\
17, \\
18, \\
19\end{array}$ & $\begin{array}{l}33.06045426, \\
33.10548665, \\
33.10548169, \\
33.10047989, \\
33.12549354, \\
33.12048705, \\
33.15549928, \\
33.14549582, \\
33.15550367, \\
33.18550949, \\
33.20051836, \\
33.20051836\end{array}$ & 33.125 & \\
\hline 2 & 9 & $\begin{array}{l}1, \\
2, \\
3, \\
4, \\
5, \\
6, \\
7, \\
8, \\
10, \\
11, \\
12, \\
13, \\
14, \\
15, \\
16, \\
17, \\
18, \\
19, \\
20\end{array}$ & $\begin{array}{c}33.06045426, \\
33.09548316, \\
33.08046737, \\
33.10548665, \\
33.10548169, \\
33.10047989, \\
33.12549354, \\
33.13049525, \\
33.14549582, \\
33.12048705, \\
33.15549928, \\
33.14549582, \\
33.15549928, \\
33.15550367, \\
33.1805119, \\
33.18550949, \\
33.20051836, \\
33.20051836, \\
33.20551995\end{array}$ & 33.125 & 33.125 \\
\hline
\end{tabular}

The snapshot of the output obtained while aggregating data at each sensor head in Table 8 shows the formation of clusters and cluster heads and how the nodes are grouped into clusters and the details of data aggregation per round.

\section{Conclusion}

It was observed that in comparison to the $\mathrm{LEACH}$ the proposed technique has,

a) Operating nodes will live longer in modified LEACH protocol as they will be functional for more rounds in comparison with LEACH protocol. 
International Journal of Distributed and Parallel Systems (IJDPS) Vol.11, No.1/2/3/4/5, September 2020

b) Operating nodes will perform better in modified LEACH protocol as they will be able to do more transmissions in comparison with LEACH protocol.

c) Energy consumption per round is less in case of modified LEACH protocol.

d) Average energy consumption is less for modified LEACH protocol in comparison to LEACH protocol.

e) Proposed Work protocol is better performing as,

- Network Life time Gain Percentage per round $=68.75 \%$

- Network Life time Gain Percentage per transmission= 58.33\%

- Average energy consumed in LEACH protocol $=0.0012 \mathrm{~J}$

- Average energy consumed in modified LEACH protocol $=0.00052 \mathrm{~J}$

- Increase in average Energy consumption Percentage $=56.667 \%$

- Network Lifetime percentage $=69.94 \%$

\section{REFERENCES}

[1] Sibanjan Das, UmitMertCakmak, Book- "Hands-On Automated Machine Learning", Packt Publishing, April 26, 2018.

[2] Pratima Sarkar and ChinmoyKar, "TH-LEACH: Threshold Value and Heterogeneous Nodes-Based Energy-Efficient LEACH Protocol”, Springer Nature Singapore Pte Ltd., 2018.

[3] S Pradhan, E Sinha, K Sharma, "Data fusion by truncation in wireless sensor network", Advanced Computational and Communication Paradigms, 544-551, 2018.

[4] N.G.Palan, B. V. Barbadekar, Suhas Patil, "A retrospective analysis of cluster head selection protocol", International Conference on Inventive Systems and Control (ICISC), 16 October 2017.

[5] MisbahLiaqat, Abdullah Gani, Mohammad Hossein Anisi, SitiHafizah Ab Hamid, Adnan Akhunzada, Muhammad Khurram Khan, Rana Liaqat Al, "Distance-Based and Low Energy Adaptive Clustering Protocol for Wireless Sensor Networks", PlosOne Publication, September 22, 2016.

[6] Sunil Dhimal, Kalpana Sharma, "Energy Conservation in Wireless Sensor Networks by Exploiting Inter-Node Data Similarity Metrics", International Journal of Energy, Information and Communications, 2015.

[7] S Pradhan, Kalpana Sharma, "Energy Efficient, Integrity Preserving and Secure Data Aggregation Technique for Wireless Sensor Network", International Journal of Security and Its Applications 9 (6), 43-52, 2015.

[8] S Pradhan, Kalpana Sharma, " Development of Technique for Formation of Equi-Sized Clusters in Wireless Sensor Networks for Efficient Enery Management", International Journal of Computer Applications 112 (14), 2015.

[9] Florence G.H. Yap, Hung-Hsu Yen, "Survey on Data Capturing/Processing/Transmission in WSN", MDPI Publications, 20 February 2014.

[10] A. S. Raval, A. Kansara, "Energy Efficient Cluster Head Selection for Data Aggregation in Wireless Sensor Networks", International Journal of Advanced Research in Computer Science and Software Engineering, Volume 4, Issue 2, February 2014.

[11] P. V. Ujawe, S. Khiani, "Review on Data Aggregation Techniques for Energy Efficiency in Wireless Sensor Networks", International Journal of Emerging Technology and Advanced Engineering, Volume 4, Issue 7, 2014.

[12] A. Tripathi, S. Gupta, B. Chourasiya, "Survey on Data Aggregation Techniques for Wireless Sensor Networks", International Journal of Advanced Research in Computer and Communication Engineering. Volume 3, Issue 7, 2014.

[13] Abubakr A. A. Alsharif, Biswajeet Pradhan, "Urban Sprawl Analysis of Tripoli Metropolitan City (Libya) Using Remote Sensing Data and Multivariate Logistic Regression Model", Journal of the Indian Society of Remote Sensing, 26 July 2013.

[14] Monica R Mundada, Savan Kiran, Shivanand Khobanna, Raja Nahusha Varsha, Seira Ann George, "A Study On Energy Efficient Routing Protocols In Wireless Sensor Networks", International Journal of Distributed and Parallel Systems (IJDPS) Vol.3, No.3, May 2012. 
[15] K.Ramesh and K.Somasundaram, "A Comparative Study Of Cluster head Selection Algorithms In Wireless Sensor Networks", International Journal of Computer Science \& Engineering Survey (IJCSES) Volume 2, Issue 4, November 2011.

[16] Waltenegus Dargie, Christian Poellabauer, "Fundamentals of Wireless Sensor Networks", WILEY Publication, January 2011.

[17] Y Li, N Yu, W Zhang, W Zhao, X You, "LEACH Protocol” ,IEEEConference on Computer Communication Worship(INFOCOM WKSHPS), 2011.

[18] V. Kumar, S. Jain, S. Tiwari, "Energy Efficient Clustering Algorithms in Wireless Sensor Networks: A Survey", IJCSI International Journal of Computer Science Issues, Volume 8, Issue 5, No 2, 2011.

[19] Yingshu Li, My T. Thai, Weili Wu, "Wireless Sensor Networks and Applications”,Springer Science + Business Media, 2008.

[20] W.R. Heinzelman, A. Chandrakasan, H. Balakrishnan, "Energy-efficient communication protocol for wireless microsensor networks", Proceedings of the 33rd Annual Hawaii International Conference on System Sciences, IEEE, 7 Jan 2000.

[21] KAGGLE repository for data.

(Dataset Link: https://www.kaggle.com/yungbyun/sensor- data).

\section{AUTHORS}

Tamal Banerjee is currently working as Software Engineer at Dell Technologies as of August, 2020. He completed schooling from Kendriya Vidyalaya with 9.2 cgpa in 10th and intermediate from Sri Chaitanya, Hyderabad with $94.15 \%$ in 12 th. He completed his B.Tech from Sikkim Manipal Institute Of Technology, Sikkim. He is always eager to learn, play and explore.

Priyansh Sharma, born in Himachal Pradesh, India. He completed schooling from S.V.M Bilaspur Himachal Pradesh with $94 \%$ in 10th and $90 \%$ in +2 . He got his Bachelor of Technology Degree in computer Science from Sikkim Manipal University. He had a great experience there and earned many new professional logics to deal with problems. Recently he worked in project named "Consensus Based Data Aggregation for Energy Conservation in Wireless Sensor Network" in Wireless Sensor network.

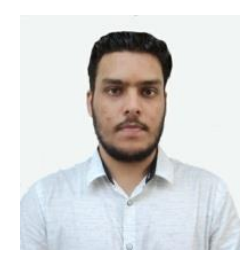

Shrijana Pradhan is currently working as Assistant Professor I in the Department of Computer Science \& Engineering at Sikkim Manipal Institute of Technology, Majhitar, Sikkim, India since August, 2011. She completed her Bachelor of Engineering in Computer Science and Engineering from Government Engineering College, Bilaspur, Chhattisgarh, India in the year 2011 with CGPA 8.01. She completed her Master of Technology in Computer Science and Engineering from Sikkim Manipal Institute of Technology, Sikkim Manipal University, Sikkim, India in

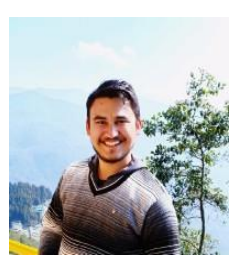
the year 2015 with CGPA 9.75 and was awarded with gold medal as university topper

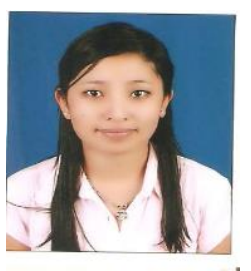
for excellence in performance in her Master of Technology programme. She has published 07 technical papers in various International Journals and conferences. She is currently pursuing her PhD in the field of Wireless Sensor Networks under the able guidance of Prof. (Dr.) Kalpana Sharma, Head, Department of CSE, SMIT. 